\title{
A master lock for deadly parasites
}

An RNA-interference screen has identified the protein CD55, expressed on the surface of red blood cells, as an essential receptor for infection of the cells by the malaria parasite Plasmodium falciparum.

\section{WAI-HONG THAM \& ALEXANDER T. KENNEDY}

$\mathrm{T}$ There are trillions of cells in the human body and around 200 distinct cell types, some of which can be hijacked as safe houses by deadly pathogens. Malaria parasites, which infect millions of humans annually, have a preference for residing in liver and blood cells. To enter these cells, the parasites make proteins that recognize other proteins on the target cell's surface. By analogy, the parasite proteins fit in a key-like fashion to their locks, the red blood cell proteins. But only a handful of these lock-and-key combinations has been discovered. Writing in Science, Egan et al. ${ }^{1}$ combine two exciting technologies RNA interference and ex vivo production of red blood cells - to identify other receptor proteins involved in malaria-parasite entry.

In 1975, live imaging of malaria parasites entering red blood cells highlighted a dynamic process with distinct observable steps $^{2}$. The form of the malaria parasite that enters these cells is a merozoite, characterized by an ovoid shape and an apical tip. After initial contact with the red blood cell, the merozoite reorients so that its apical tip is in close proximity to the cell surface. Parasite proteins in the apical prominence recognize red blood cell proteins, triggering irreversible commitment to entry - turning the key in the lock and opening the door. Subsequently, a tight junction forms between parasite and red blood cell membranes, propelling the parasite into the cell and beginning the blood stage of infection. Intensive efforts to develop a vaccine against this stage have focused on identifying all the lock-and-key combinations involved in parasite invasion and on ways to block parasite entry.

Egan et al. have performed the first large knockdown screen of red blood cell proteins that are bound by the most lethal human malaria parasite, Plasmodium falciparum. The researchers targeted 42 genes that encode proteins determining blood group, on the basis that all $P$. falciparum receptors known so far belong to this protein group and that these genes are not involved in red blood cell production (Fig. 1). The authors inserted a library of small hairpin RNA molecules (shRNAs) that bind to, and thus inhibit expression of, these genes into isolated haematopoietic progenitor cells (which give rise to all blood cells), and then induced these cells to proliferate and differentiate into red blood cells. Once the cells were mature enough to sustain parasite development, they were infected with P. falciparum expressing green fluorescent protein, thus allowing infected cells to be identified.

The authors then sequenced the shRNAs in these cells and compared the levels of each shRNA between infected and non-infected cell populations. Positive targets were identified as genes whose corresponding shRNAs were under-represented in the infected population, following the logic that inhibiting these genes impairs parasite infection. Among the authors' positive hits were genes encoding the proteins basigin and complement receptor 1 (CR1), which are known to be involved in P. falciparum invasion ${ }^{3,4}$. More excitingly, they identified the cell-surface protein CD55 as the top-ranked candidate for a new entry portal for P. falciparum.

A key aspect of this discovery is that CD55 is an essential lock in the invasion process: Egan and colleagues show that diverse P. falciparum laboratory strains and field isolates could not infect red blood cells lacking CD55. The parasite uses several lock-and-key combinations to enter red blood cells, to maximize its opportunities for entry ${ }^{5}$, and this redundancy presents a huge hurdle to the development of vaccines to block blood-stage infection. Therefore, interactions that are essential in the invasion process provide attractive vaccine candidates. The effect of loss of CD55 on parasite invasion is similar to the loss of basigin, whose parasite protein partner PfRh5 is a leading blood-stage vaccine candidate ${ }^{6}$. Clearly, an outstanding question stemming from this work is the identification of the $P$. falciparum protein that binds to CD55. If CD55 is a crucial host factor for P. falciparum invasion, the hypothesis would be that the parasite ligand is also an essential factor and thus warrants inclusion in the vaccine-development pipeline.

Egan et al. also show that loss of CD55 on red blood cells affects the proliferation of diverse P. falciparum strains. The authors suggest that CD55 may be involved in the a

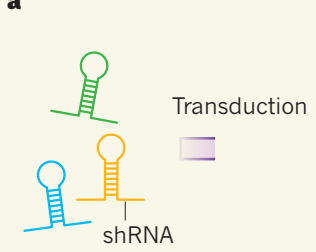

HPC

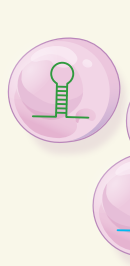

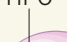
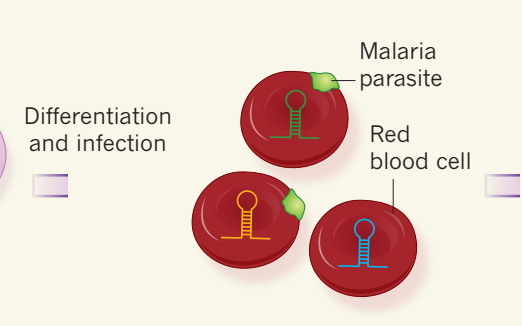

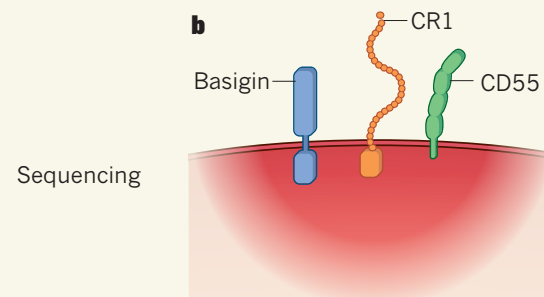

Figure 1 | RNA interference identifies malaria-parasite entry receptors. a, To identify proteins on red blood cells to which malaria parasites bind, Egan et al. ${ }^{1}$ created a library of short hairpin RNA (shRNA) molecules that block the expression of genes encoding blood-group proteins. These shRNAs were transduced into haematopoietic progenitor cells (HPCs), and the HPCs were induced to proliferate and differentiate into red blood cells. The authors then infected the cells with fluorescent malaria parasites and compared the abundance of shRNA molecules in uninfected and infected cells; those shRNAs that were under-represented in the infected population were determined to correspond to genes encoding receptor proteins for parasite entry, because inhibition of these genes would inhibit the parasite's ability to infect. b, Alongside the known parasite receptors CR1 and basigin, the authors identified several new candidates, of which CD55 was the top hit. 
irreversible-commitment phase of parasite invasion. This hypothesis should be further explored using live and high-resolution imaging of merozoites attempting to infect CD55deficient red blood cells. It will be interesting to determine whether the loss of CD55 affects the establishment of commitment, deformation of the red blood cell surface or signalling for tight-junction formation during the early stages of parasite invasion.

Could CD55 be a therapeutic target for malaria infections? Although some healthy humans exist without CD55 on their blood cells, important caveats arise for individuals living in malarious regions. Field studies show a correlation between declining CD55 levels on red blood cells and severe malarial anaemia, potentially due to the destruction of red blood cells by the complement system ${ }^{7}$, an arm of the immune system that is regulated by CD55. An alternative avenue could be to explore the potential of soluble CD55 as a competitor to red blood cell CD55 for merozoite binding, and thus an inhibitor of parasite growth.

An emerging theme in P. falciparum invasion is that the parasite exploits complement regulators as entry receptors. In humans, CR1 and CD55 protect self-tissues from complement attack. A variable currently missing from experimental work monitoring $P$. falciparum invasion is the addition of active complement-system components during parasite entry. For example, complement activation is known to modulate the behaviour of both CD55 and CR1 on membranes, resulting in changes in red blood cell deformability that may affect merozoite entry ${ }^{8,9}$. Binding of parasite ligands to these receptors may interfere with their regulatory roles (as is the case with CR1), and the consequences of this for red blood cell survival during infection need to be understood. Current research has led to a remarkable deconstruction of the distinct steps in $P$. falciparum invasion ${ }^{10}$, but future challenges will be to understand merozoite entry in the context of complement activation and immune attack.

Wai-Hong Tham and Alexander T. Kennedy are at the Walter and Eliza Hall Institute, Parkville, Victoria 3052, Australia, and the Department of Medical Biology, University of Melbourne, Parkville. e-mail:tham@wehi.edu.au

1. Egan, E. S. et al. Science 348, 711-714 (2015)

2. Dvorak, J. A., Miller, L. H., Whitehouse, W. C. \& Shiroishi, T. Science 187, 748-750 (1975).

3. Tham, W.-H. et al. Proc. Natl Acad. Sci. USA 107, 17327-17332 (2010)

4. Crosnier, C. et al. Nature 480, 534-537 (2011).

5. Cowman, A. F. \& Crabb, B. S. Cell 124, 755-766 (2006).

6. Douglas, A. D. et al. Cell Host Microbe 17, 130-139 (2015).

7. Biryukov, S. \& Stoute, J. A. Trends Mol. Med. 20, 293-301 (2014)

8. Glodek, A. M. et al. Blood 116, 6063-6071 (2010).

9. Karnchanaphanurach, P. et al. J. Clin. Invest. 119, 788-801 (2009)

10.Riglar, D. T. et al. Cell Host Microbe 9, 9-20 (2011).

\section{CELL BIOLOGY}

\section{Nuclear dilemma resolved}

\section{After cell division, membranes become fused around the nucleus to encapsulate the cell's chromosomes. It emerges that this process is regulated by the ESCRT-III protein complex. SEE LETTERS P. $231 \&$ P. 236}

\section{BRIAN BURKE}

I The chromosomes of animal, plant and fungal cells are enclosed within a nucleus, which is encapsulated by a membranous structure called the nuclear envelope. This poses a problem when the time comes for cells to divide, because chromosomes segregate into two daughter cells by binding to and moving along a structure called the mitotic spindle, which, at least in multicellular organisms, is located outside the nucleus. In vertebrate cells, the nuclear envelope is normally partly or completely disassembled before chromosome segregation, and new envelopes are assembled afterwards - a process that requires extensive membrane fusion. However, the mechanisms by which membrane fusion occurs have long puzzled cell biologists. In this issue, Olmos et al. ${ }^{1}$ (page 236) and Vietri et al. ${ }^{2}$ (page 231) reveal that the nuclear envelope co-opts a membrane-sculpting protein complex called ESCRT-III to bring about reassembly.

The nuclear envelope completely encloses an
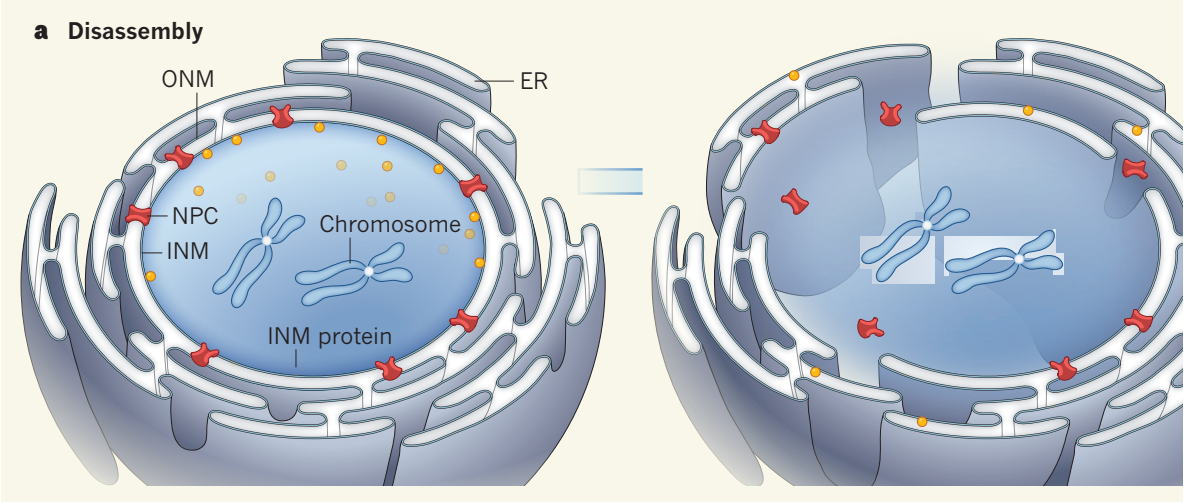

b Reassembly
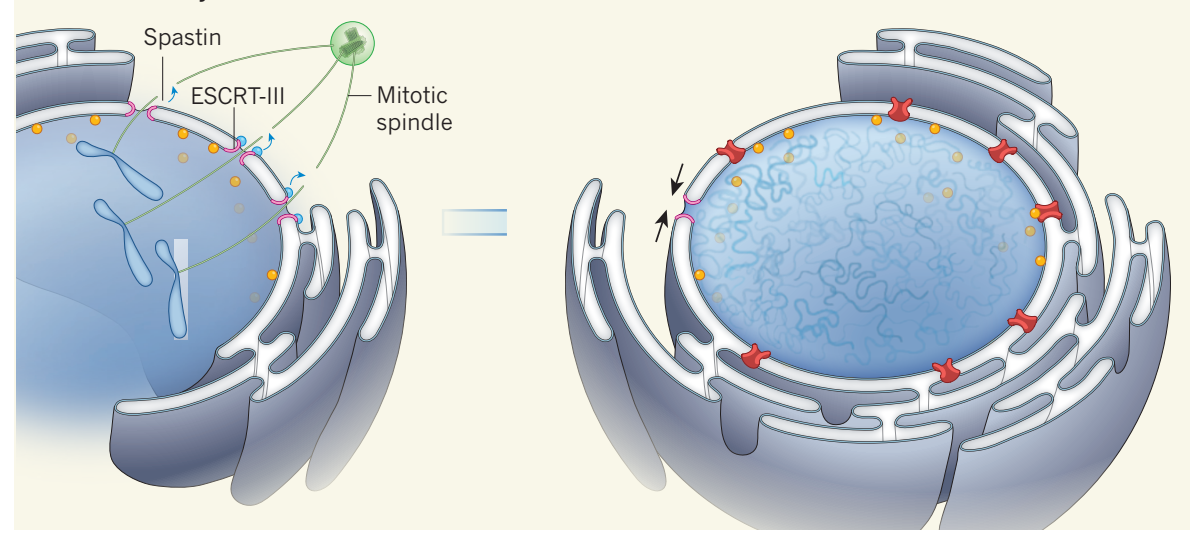

Figure 1 | Resealing the nuclear envelope. a, The nuclear envelope is composed of inner and outer nuclear membranes (INM and ONM, respectively). The two are joined at junctions filled by nuclear pore complexes (NPCs), and the ONM is joined to another membranous structure, the endoplasmic reticulum (ER). During cell division, the nuclear envelope disassembles, and INM proteins disperse into the ER. b, As the cell divides, chromosomes (which separate and then become decondensed) are pulled to opposite poles of the cell by a structure called the mitotic spindle. Vietri et al. ${ }^{2}$ demonstrate that the ESCRT-III protein complex recruits the enzyme spastin to sever the mitotic spindle. Both this group and Olmos et al. ${ }^{1}$ show that ESCRT-III then promotes resealing of the nuclear envelope (black arrows). 